\title{
Learning fuzzy rules to characterize objects of interest from remote sensing images
}

\author{
Bruno Belarte ${ }^{1}$, Cédric Wemmert ${ }^{1}$, Germain Forestier ${ }^{2}$, Manuel Grizonnet $^{3}$, Christiane Weber $^{4}$ \\ ${ }^{1}$ Université de Strasbourg ICube - UMR 7357, CNRS, \{belarte,wemmert\}@ unistra.fr \\ ${ }^{2}$ Université de Haute-Alsace, MIPS - EA 2332, France, germain.forestier@uha.fr \\ 3 CNES, DCT/SI/AP, 18, avenue Edouard Belin, 31401 Toulouse Cedex 09, manuel.grizonnet@cnes.fr \\ ${ }^{4}$ Université de Strasbourg, LIVE - ERL 7230, CNRS christiane.weber@live-cnrs.unistra.fr
}

\begin{abstract}
In this article a new method for learning concepts from examples of objects provided by experts for remote sensing images is presented. The goal of this method is to give the geographer expert a description of complex objects of interest extracted from very high resolution remote sensing images. The description of such objects needs to handle imprecision inherent to segmentation and very high resolution images. The first step of this approach is to classify objects composing all the examples. This classification allows the learning of a rule describing how the examples are composed regarding the segmentation. Finally, this rule is used to extract objects corresponding to the examples. Experiments on a remote sensing image of a urban landscape in Toulouse, France are presented to show the relevance of the method.
\end{abstract}

\section{INTRODUCTION}

The technological evolution in the acquisition of remote sensing images gives us access to images with a high level of detail. This is even more true with Very High Resolution (VHR) remote sensing images acquired by recent satellites such as QUICKBIRD (C DigitalGlobe 2001) and PLÉIADES (C) CNES 2012). In order to analyze such images, object oriented methods have been developed. An image is segmented into regions or objects (aggregates of homogeneous pixels). Such approaches make it possible to compute structural and radiometric properties on these objects rather than consider only the pixel spectral values. Moreover, spatial relations between objects such as adjacency and distance can be computed, enhancing the properties available for objects. A geographer expert can describe an object of interest regarding these properties. Such a description of an objects class is called a concept. These concepts can then be used to help to classify a segmented image.

Urban landscapes are extremely complex in essence because they are man-made: objects such as roads and buildings can be composed of heterogeneous radiometric values and have regular shapes (rectangle, polygon, ...), that unfortunately cannot be precisely extracted by a segmentation algorithm. The expert can describe the concept house but only a few objects in the scene will match this concept because of the over-segmentation of a house that can be split into one or more parts, depending on its orientation to the sun or the complexity of the shape of the house. An example of both over- and under-segmentation is visible in figure 2(c). Such limitations make it very difficult to use expert knowledge in urban landscape image analysis. Methods extracting particular objects of interest (building or road detection algorithms) exist. However, these are often limited to a given type of image (panchromatic, multi-spectral, SAR, ...) and/or a given resolution.

In this paper we propose a new method for learning concepts from examples. The knowledge representation and the reasoning algorithm used in the process are based on fuzzy logic. Fuzzy logic has already being successfully used in remote sensing image interpretation. [1] uses fuzzy logic classification on a multi scale segmentation to extract and monitor urban vegetation. [2] uses fuzzy logic and morphological filtering to characterize roofless buildings. [3] uses OBIA and a fuzzy rules base for objects extraction from VHR satellite images. We illustrate this approach of learning the concept of house on a QUICKBIRD image. The method proposed is generic and thus can be used on any kind of image as long as the objects of interest are discernible in the image.

\section{Methodology}

In this section, we present the method developed for learning concepts from examples given by a geographer expert. Let us give some definitions. A constraint is an interval of values that a property should match. In order to handle the imprecise nature of segmentation and image analysis, these constraints are formalized by fuzzy sets. Fuzzy sets are combined into fuzzy rules allowing the use of a fuzzy reasoning algorithm [4]. A concept is defined by a fuzzy rule and has a label that will be used to classify objects. Concepts are ordered in a hierarchy of concepts. Concepts of a higher level of abstraction, describing complex objects composed of several smaller objects, are called composite concepts. Concepts and hierarchy compose the knowledge base. An elementary object is an object produced by the segmentation algorithm. Such an object is described by its geometric shape (a polygon) and by three kinds of properties: attributes (radiometric and structural, such as size, elongation or NDVI value), spatial relations (such as adjacency or distance) and composition relations. The geographer expert can define an example of an object of interest by means of the polygon representing its bounding (figure 2(b)). Elementary objects matching these examples, 


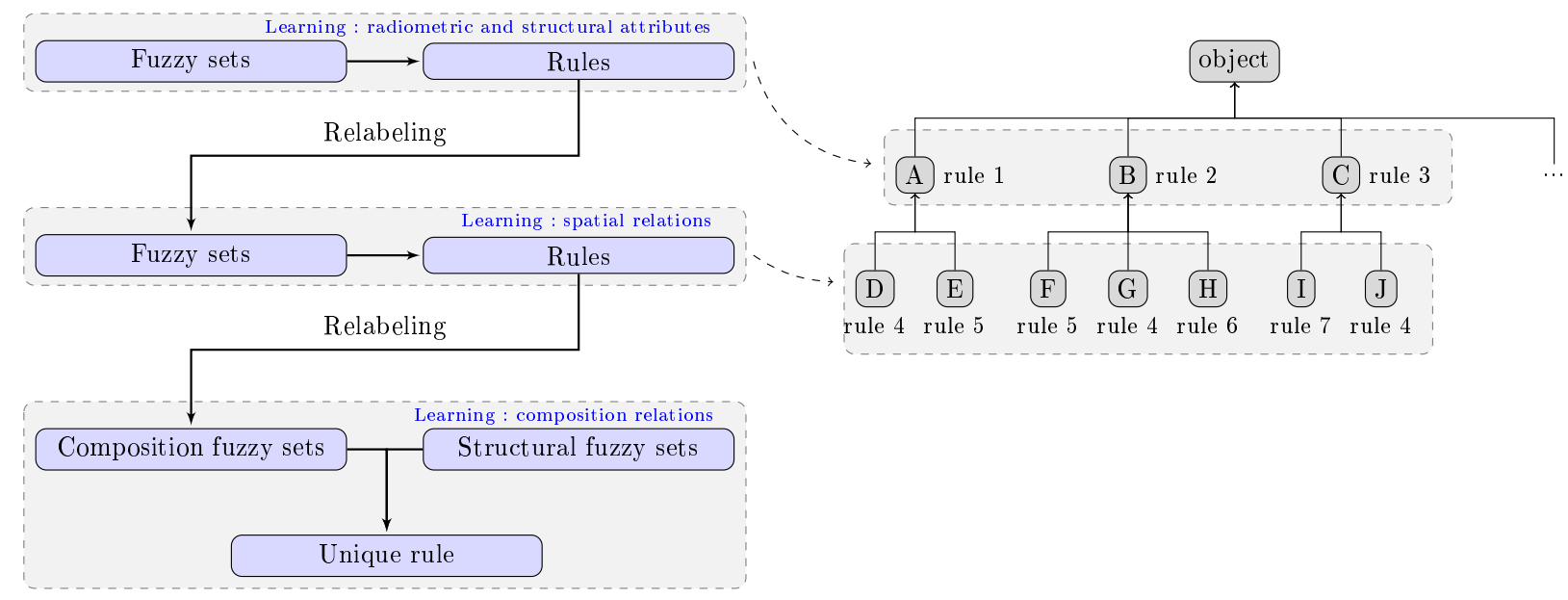

Fig. 1: Flowchart of the learning process. On the left is the different learning steps. On the right, the corresponding generated layers in the hierarchy of concepts is shown.

thus corresponding to a part of an example, are called example objects.

The proposed method aims at learning fuzzy sets and combining them into fuzzy rules to produce new concepts. The input image is preprocessed by a segmentation algorithm to produce an adjacency graph (nodes corresponding to elementary objects and arcs corresponding to adjacency relations between two objects). Due to space constraint of the article, the graph is not represented. Each object is labeled by its corresponding concept from the hierarchy, computed by a fuzzy reasoning algorithm [4]. If an object does not match any concepts in the knowledge base, it keeps the label object. Then, objects corresponding to the examples given by the expert are extracted. An example could correspond to one object or several, depending on the level of the over-segmentation.

A learning step consists of an ascendant hierarchical clustering (AHC [5]) on properties (for sets) or objects (for rules). At the beginning, each object defines a new cluster and the two closest clusters are merged while the stopping criterion is not reached. To be as automatic as possible, the stopping criterion chosen is the Ward dissimilarity measure [6]. When the increase of inter clusters inertia is greater than a given threshold, the two closest clusters are not merged and the AHC stops. Figure 1 illustrates the different steps of the learning process. First, the fuzzy sets of the object properties are learned. These fuzzy sets are used to characterize the examples. For each example, an array filled with return values of each fuzzy set is created. These arrays are then classified with the same method. This classification produce rules, one for each class. Each rule defines a concept which will enrich the first layer in the hierarchy, thus refining the concept object. The generated concepts are used to label the example objects with a fuzzy reasoning algorithm. This step makes it possible to learn the spatial relations between objects. A new set of fuzzy sets, based only on spatial relations is learned and these new fuzzy sets are used to learn new rules. Each rule on spatial relations defines one or several concepts in the hierarchy, depending on how it refines a previously defined concept (second layer in the hierarchy). For example, some objects labeled $A$ or $B$ (but not all of them) match the rule 5 in Figure 1 so the rule 5 will define two concepts as descendants of both concepts $A$ and $B$. The concepts generated by the learning algorithm are then used to relabel the example objects. The final step is the learning of the composition fuzzy sets in addition to structural fuzzy sets on the examples. This step produces a single composition rule describing the composite concept corresponding to the class of the given examples.

The composition rule can be used to extract composite objects from the graph. This is done by a composite objects extraction process based on a region growing algorithm [7] adapted to a graph of objects. A composite concept is described by a composition rule. For example, the composite concept residential building block is defined by the rule composed of houses and composed of vegetation and surrounded by road. Each object with such a label (after relabeling the whole graph) is a candidate seed for the region growing algorithm. For all seeds, a region growing takes place and a composite object is created from this region. If this object satisfies the composition rule, it is added to the graph.

\section{RESULTS}

In this experiment we focused on extracting houses from a residential area. The image used is a QUICKBIRD image acquired in 2002 of an area near Toulouse, France, with a resolution of approximately 0.7 meters. As stated in section I the segmentation of such a VHR image can only result in over-segmentation. As a consequence we consider houses as complex objects and our purpose is to learn the composition rule describing this concept. As urban buildings are partly defined by their surrounding, we chose to use adjacency to 


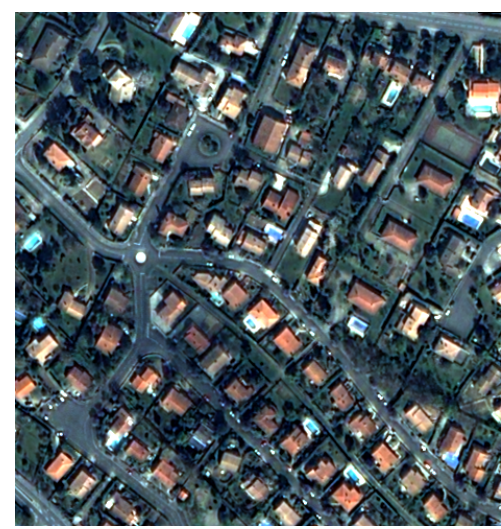

(a) The raw image, QUICKBIRD from Toulouse, France

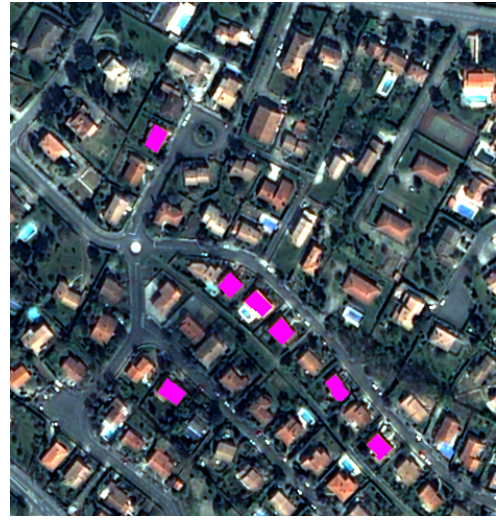

(b) Examples provided by the expert

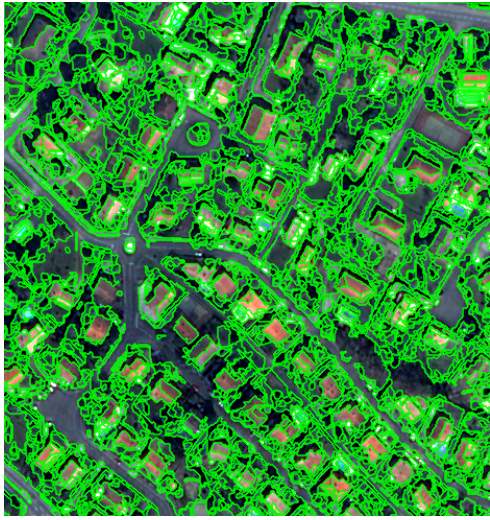

(c) The segmentation of the image. Green polygons correspond to the bounding of each segment

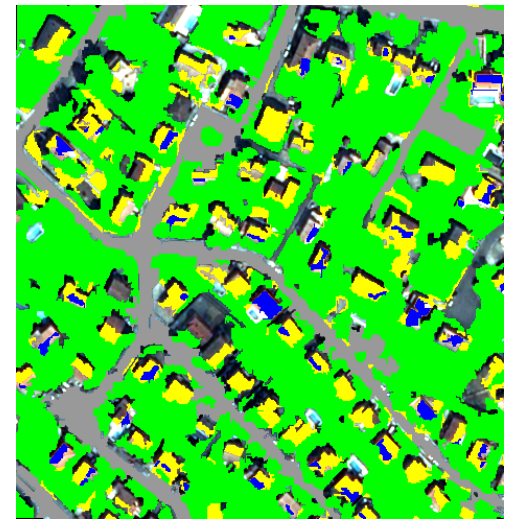

(d) Concepts learned by the method, two concepts were learned (blue and yellow).

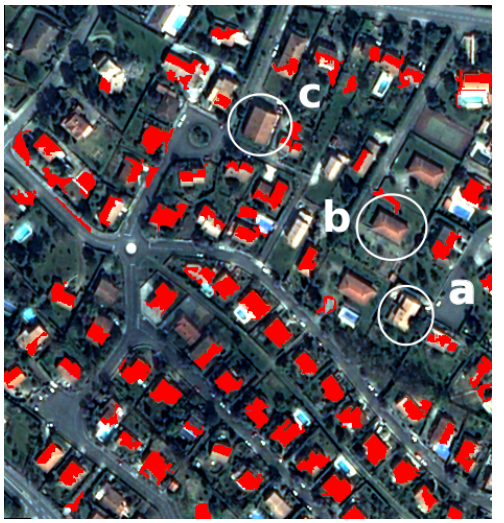

(e) Extracted houses in red

Fig. 2: Image and examples used in the experiments.

vegetation and roads to help us characterize houses. Figure 2(a) shows the raw image and examples provided by the geographer expert are shown in Figure 2(b). We used the Meanshift algorithm to obtain a segmentation of the image (an example of segmentation is given in figure 2(c)).

After the segmentation step, vegetation and road objects are labeled. Vegetation objects are labeled with a fuzzy reasoning engine and a rule provided by the expert, road objects are labeled with the help of a geographic information system. This allows us to calculate the adjacency to vegetation and the distance to road. Then, the learning process takes place. Example objects are extracted, fuzzy sets and fuzzy rules are learnt, concepts are created from these rules, a composition rule is learnt and the composite concept corresponding to the examples is generated. These learned concepts are used to label objects in the graph. Figure 2(d) shows that two concepts have been learnt in this example. Finally, the composite concept is used to extract houses from the image (figure 2(e)). Houses are identified with a precision of 0.82 according to the ground truth provided by the geographer expert.

Additional experiments were made to evaluate the influence of the segmentation and learning parameters. The Meanshift segmentation has been applied several times with a large range of spectral and spatial parameters and the learning process has been run several times with a large range of thresholds for the Ward criterion (for sets and rules). Results show that the segmentation step is still critical. The results given in figure 2 were obtained using the best parameters found during this study.

Houses are better identified when the image is only slightly over-segmented. But in such case roads and vegetation areas are under-segmented and the extraction of composite objects of a higher level of abstraction (urban blocks for instance) is then compromised.

\section{REFERENCES}

[1] C.-Q. Ke, L. Xia, G.-D. Tang, and X. Cao, "Detecting urban vegetation using a object-oriented method with quickbird imagery," vol. 3, july 2008 , pp. III-138-III-141.

[2] L. Gueguen, M. Pesaresi, A. Gerhardinger, and P. Soille, "Characterizing and counting roofless buildings in very high resolution optical images," Geoscience and Remote Sensing Letters, IEEE, vol. 9, no. 1, pp. 114-118, jan. 2012. 
[3] I. Sebari and D.-C. He, "Automatic fuzzy object-based analysis of VHSR images for urban objects extraction," ISPRS Journal of Photogrammetry and Remote Sensing, vol. 79, pp. 171-184, May 2013.

[4] L. Zadeh, "Fuzzy logic and approximate reasoning," Synthese, vol. 30, no. 3, pp. 407-428, 1975.

[5] J. Hartigan, Clustering algorithms, ser. Wiley series in probability and mathematical statistics: Applied probability and statistics. Wiley, 1975.
[6] J. H. Ward, "Hierarchical grouping to optimize an objective function," Journal of the American Statistical Association, vol. 58, no. 301, pp. 236-244, 1963.

[7] R. Adams and L. Bischof, "Seeded region growing," IEEE Transactions on Pattern Analysis and Machine Intelligence, vol. 16, no. 6, pp. 641-647, Jun. 1994. 